\title{
MicroRNA-520c inhibits glioma cell migration and invasion by the suppression of transforming growth factor- $\beta$ receptor type 2
}

\author{
SHENGLI HU $^{1 *}$, HUIBIN CHEN ${ }^{2 *}$, YUQIANG ZHANG ${ }^{1}$, CHAOJIA WANG $^{1}$, \\ KAIJUN LIU ${ }^{1}$, HUI WANG ${ }^{1}$ and JIE LUO ${ }^{1}$ \\ ${ }^{1}$ Department of Neurosurgery, Taihe Hospital, Hubei University of Medicine, Shiyan, Hubei 442000; \\ ${ }^{2}$ Department of Surgery, The First People's Hospital of Qujing City, Qujing, Yunnan 655000, P.R. China
}

Received July 25, 2016; Accepted September 9, 2016

DOI: $10.3892 / o r .2017 .5421$

\begin{abstract}
Dysregulation of microRNAs (miRNAs) is actively involved in the development and progression of glioma. miR-520c was previously found to inhibit glioblastoma cell migration. However, the clinical significance of miR-520c and its biological function in glioma remain largely unknown. In the present study, we found that miR-520c expression in glioma tissues was significantly decreased compared to adjacent non-cancerous tissues. The low level of miR-520c was prominently correlated with advanced World Health Organization (WHO) grade and decreased overall survival of glioma patients. Overexpression of miR-520c in U251 cells significantly decreased the migration and invasion of the cancer cells, while miR-520c silencing promoted U87 cell migration and invasion in vitro. Mechanistically, miR-520c inversely regulated transforming growth factor- $\beta$ receptor type 2 (TGFBRII) abundance in the glioma cells. Herein, TGFBRII was found to be a downstream target of miR-520c in glioma. Furthermore, an inverse correlation between TGFBRII and miR-520c expression was observed in the glioma cases. In constrast, restoration of TGFBRII expression abrogated the effects of miR-520c overexpression in U251 cells with increased cell migration and invasion. In addition, miR-520c overexpression blocked TGF- $\beta 1$-induced cell migration and invasion in U251 cells. Collectively, miR-520c may serve as a prognostic predictor and a therapeutic target for glioma patients.
\end{abstract}

\section{Introduction}

MicroRNAs (miRNAs), a group of small non-coding RNAs interacting with the 3'-untranslated region (3'-UTR) of targeted

Correspondence to: Dr Shengli Hu, Department of Neurosurgery, Taihe Hospital, Hubei University of Medicine, 32 People South Road, Shiyan, Hubei 442000, P.R. China

E-mail: hushengli_shiyan@163.com

${ }^{*}$ Contributed equally

Key words: microRNA-520c, glioma, cell migration, invasion, TGFBRII
mRNAs, inhibit the expression of target genes by contributing to the degradation or translational inhibition of target mRNAs (1). They have been found to be actively involved in various biological processes including cell proliferation, apoptosis, differentiation and movement $(2,3)$. Emerging studies have shown that abnormal expression and function of miRNAs play important roles in the initiation and progression of human malignancies (4-6). In addition, miRNAs have been demonstrated to be promising biomarkers and therapeutic targets of glioma (7-9). Investigating the expression and biological function of miRNAs in glioma may contribute to the identification of novel biomarkers and therapeutic targets for glioma patients.

Recently, microRNA-520c (miR-520c) was found to play important roles in various human cancers including diffuse large B cell lymphoma (10), breast cancer (11), fibrosarcoma (12) and hepatocellular carcinoma (HCC) (13). A study on breast cancer showed that miR-520c promoted cancer cell migration and invasion in vitro and in vivo by suppression of CD44 (14). In addition, expression of miR-520c increased proliferation, migration and invasion of HCC cells in vitro (13). However, miR-520c abrogated both in vitro cell invasion and in vivo intravasation of highly invasive MDA-MB-231 cells (11), indicating that miR-520c functions as a tumor-suppressor in estrogen receptor negative breast cancer. Previously, Lu et al reported that miR-520c inhibits glioblastoma U87GM cell migration with decreased activation of proMMP2 in vitro (15). However, the clinical significance and biological role of miR-520c in glioma remain practically unknown.

In the present study, we confirmed that the expression of miR-520c was decreased in glioma tissues. Low expression of miR-520c was correlated with poor clinicopathological features and decreased survival of glioma patients. Our data showed that miR-520c inhibited the metastatic ability of glioma cells in vitro. Moreover, transforming growth factor- $\beta$ receptor type 2 (TGFBRII) was identified as a downstream target of miR-520c in glioma.

\section{Materials and methods}

Clinical tissues. Fresh human glioma and adjacent non-cancerous tissues were obtained from 60 patients who underwent therapeutic removal of gliomas at Taihe Hospital, Hubei University of Medicine. Patients did not receive any 
chemotherapy or radiotherapy before surgical treatment. All clinical specimens were collected and used after obtaining informed consent from each patient enrolled in the present study. All specimens were stored in liquid nitrogen for further investigation. The protocol which involved clinical specimens in the present study was approved by the Research Ethics Committee of Hubei University of Medicine.

Cell culture and transfection. Human glioma cell lines including U87 and U251, and human kidney epithelial cell line (293T) were obtained from the American Type Culture Collection (ATCC; Manassas, VA, USA). These cells were cultured in Dulbecco's modified Eagle's medium (DMEM) with $10 \%$ fetal bovine serum (FBS), $100 \mathrm{U} / \mathrm{ml}$ penicillin and $100 \mathrm{mg} / \mathrm{ml}$ streptomycin. All cell cultures were maintained in a humidified cell incubator with $5 \% \mathrm{CO}_{2}$ at $37^{\circ} \mathrm{C}$.

miR-520c and negative control (NC) mimics, miR-520c and NC inhibitors (anti-NC) were obtained from Genecopoeia (Guangzhou, China), and were then transfected into glioma cells with Lipofectamine 2000 following the manufacturer's protocol. Retroviral vectors pMMP-TGFBRII were generated by inserting the TGFBRII cDNA into pMMP. Retrovirus packaging and transduction were previously described (16).

Quantitative real-time RT-PCR ( $q R T-P C R$ ). Total RNA from glioma cells was extracted by miRNeasy Mini kit (Qiagen, Hilden, Germany) and total RNA from glioma tissues was extracted with TRIzol reagent (Ambion, Thermo Scientific, Shanghai, China). miR-520c levels in these samples were assayed using TaqMan MicroRNA assays based on the manufacturer's protocol (Applied Biosystems, Carlsbad, CA, USA). Real-time PCR of TGFBRII was performed using an UltraSYBR Mixture (CW0957; CWBIO, Beijing, China) and an LC480 PCR System (Roche, Indianapolis, IN, USA). The primers for miR-520c and U6, TGFBRII and GAPDH were obtained from Genecopoeia. The expression levels of mRNA and miR-520c were normalized to the endogenous controls GAPDH and U6 respectively, using the $2^{-\Delta \Delta \mathrm{Ct}}$ method.

Luciferase reporter assay. To investigate whether miR-520c interacted with the 3'-UTRs of TGFBRII, wild-type (wt) 3'-UTR of TGFBRII predicted to interact with miR-520c was amplified. Then, the wt $3^{\prime}$-UTR of TGFBRII and the corresponding vectors $(\mathrm{NC}, \mathrm{miR}-520 \mathrm{c}$ mimic, anti-NC, miR-520c inhibitor or mutant miR-520c) were co-transfected into $293 \mathrm{~T}$ cells by Lipofectamine 2000. Forty-eight hours after co-transfection, the cells were lysed and assayed using Dual-Luciferase ${ }^{\circledR}$ Reporter Assay kit (Promega, Madison, WI, USA) based on the manufacturer's instructions.

Wound healing assay. Glioma cells transfected with the corresponding vectors were seeded in 6-well plates to form a single confluent cell layer. Wounds were made in the confluent cell layer using $100-\mu 1$ tips. Following wound scratching (0 and $12 \mathrm{~h}$ ), the width of the wounds were photographed with a phase-contrast microscope.

Migration and invasion assays. The migratory and invasive abilities of glioma cells were evaluated with Transwell chambers (BD Biosciences, Franklin Lakes, NJ, USA). Glioma cells $\left(5-10 \times 10^{4}\right)$ suspended in $100 \mu 1$ serum-free medium were seeded into the upper chamber, and the lower chamber was filled with $20 \%$ FBS to induce glioma cell migration or invasion through the membrane. Matrigel (1:6 dilution; Becton-Dickinson Labware, Bedford, MA, USA) was added to the upper chamber for the invasion assay. Twenty-four hours later, cells that migrated or invaded the Transwell membrane were stained with crystal violet and the number of cells was counted under a microscope. TGF- $\beta 1(2.5 \mathrm{ng} / \mathrm{ml}$; R\&D Systems, Minneapolis, MN, USA) was used for inducing glioma cell migration and invasion.

Western blotting. Before protein extraction, glioma cells were washed with phosphate-buffered saline (PBS) to remove the culture media. Cellular proteins were obtained from glioma cells using RIPA lysis buffer and the protein concentrations were assessed using the BSA method. Cellular proteins ( $30 \mu \mathrm{g})$ were separated by $10 \%$ SDS-PAGE, and were transferred to polyvinylidene fluoride (PVDF) membranes (Millipore, Bedford, MA, USA). After blocking with 5\% non-fat milk at room temperature for $1 \mathrm{~h}$, the membranes were probed with primary antibodies at $4^{\circ} \mathrm{C}$ overnight. Then, the membranes were incubated with the corresponding secondary antibodies at room temperature for $2 \mathrm{~h}$. The primary antibodies used in the present study included: TGFBRII (K105) (\#3713; Cell Signaling Technology, Danvers, MA, USA), CD44 (WL0140; Wanleibio, Shanghai, China) and GAPDH (G8140; US Biological, Swampscott, MA, USA).

Immunohistochemistry (IHC). Before IHC staining, glioma tissues were fixed with $4 \%$ formalin and embedded with paraffin. Then, the embedded tissues were cut into 4- $\mu \mathrm{m}$ thick sections and visualized by IHC staining following the standard protocol in order to evaluate the expression level of TGFBRII (Cell Signaling Technology) in glioma tissues.

Statistical analysis. All quantitative data are presented as the mean \pm the standard error of the mean (SEM). Statistical analyses including Pearson Chi-squared test, a two-tailed Student's t-test, Kaplan-Meier method, the log-rank test and Spearman's correlation analysis were performed with GraphPad Prism 6 (GraphPad Software Inc., San Diego, CA, USA). P $<0.05$ was considered to indicate a statistically significant result.

\section{Results}

Clinical significance of miR-520c expression in glioma specimens. To examine the expression status of miR-520c in glioma, qRT-PCR was performed for 60 pairs of glioma and adjacent non-tumor tissues. Our results showed that glioma tissues had significant decreased expression levels of miR-520c compared with adjacent non-tumor tissues $(\mathrm{P}<0.05$; Fig. 1A). To clarify the clinical significance and prognostic value of miR-520c in glioma, all patients were divided into two groups (miR-520c low-expressing and miR-520c highexpressing groups) according to the median level of miR-520c expression. As shown in Table I, patients with low expression of miR-520c had advanced World Health Organization (WHO) stage $(\mathrm{P}=0.024)$. Furthermore, Kaplan-Meier analysis showed that patients with low expression of miR-520c exhibited 

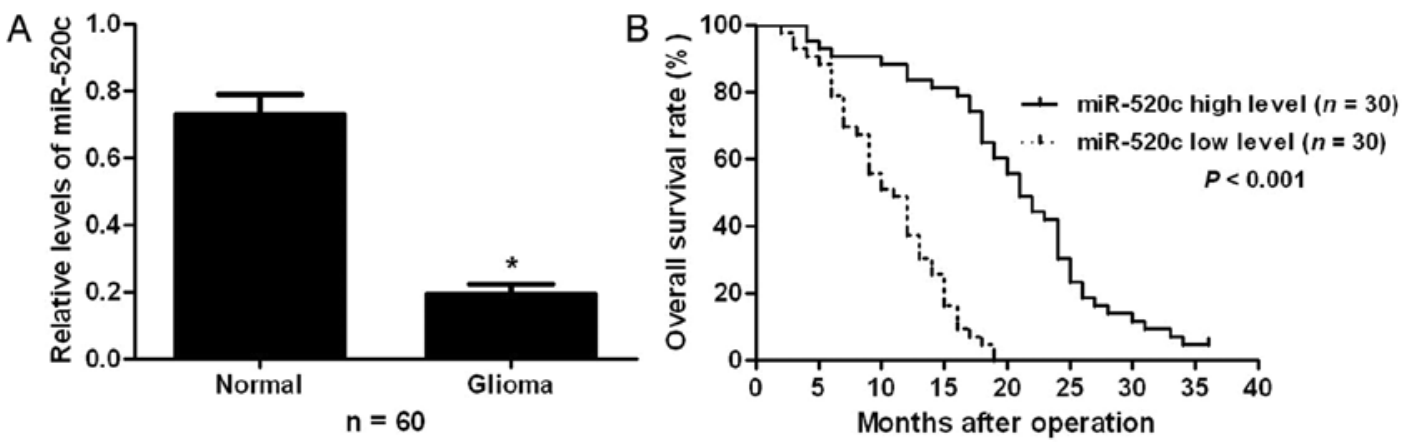

Figure 1. Expression and prognostic value of miR-520c in glioma. (A) Significantly decreased expression of miR-520c was observed in glioma tissues compared to adjacent non-cancerous tissues; ${ }^{*} \mathrm{P}<0.05$. (B) Compared with the patients with high miR-520c expression level, patients with low miR-520c expression had significantly decreased overall survival.

Table I. Correlation between the clinicopathological characteristics and expression of miR-520c in gliomas.

\begin{tabular}{lcccc}
\hline & & \multicolumn{2}{c}{ No. of patients } & \\
\cline { 3 - 4 } & $\begin{array}{c}\text { Total no. of } \\
\text { pts. (n=60) }\end{array}$ & $\begin{array}{c}\text { Low } \\
\text { miR-520c }\end{array}$ & $\begin{array}{c}\text { High } \\
\text { miR-520c }\end{array}$ & P-value \\
\hline Age (years) & & & & 0.301 \\
$<50$ & 28 & 12 & 16 & \\
$\geq 50$ & 32 & 18 & 14 & \\
Gender & & & & 0.592 \\
Male & 38 & 18 & 20 & \\
Female & 22 & 12 & 10 & \\
Tumor size (cm) & & & & 0.417 \\
$<5$ & 21 & 9 & 12 & \\
$\geq 5$ & 39 & 21 & 18 & \\
Histologic tumor & & & & 0.698 \\
type & & & & \\
Astrocytic & 42 & 22 & 20 & \\
Oligodendrogial & 7 & 3 & 4 & \\
$\quad$ Oligoastrocytic & 11 & 5 & 6 & \\
WHO grade & & & & $0.024^{\mathrm{a}}$ \\
I+II & 18 & 5 & 13 & \\
III+IV & 42 & 25 & 17 & \\
\hline
\end{tabular}

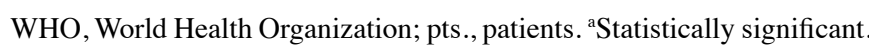

a significantly decreased overall survival rate $(\mathrm{P}<0.001$; Fig. 1B). These results indicate that miR-520c probably plays an oncogenic role in glioma.

miR-520c inhibits the migration and invasion of glioma cells. Next, we explored whether miR-520c modulates the migration and invasion of glioma cells. Transfection of miR-520c mimic into U251 cells significantly increased the expression level of miR-520c ( $\mathrm{P}<0.05$; Fig. 2A). The wound healing assays showed that the migration of U251 cells was significantly decreased after miR-520c overexpression ( $\mathrm{P}<0.05$; Fig. 2B). In addition, Transwell assays demonstrated that overexpression of miR-520c prominently suppressed the migration and invasion of $\mathrm{U} 251$ cells $(\mathrm{P}<0.05$, respectively; Fig. $2 \mathrm{C})$. In contrast, miR-520c inhibitor significantly decreased the expression level of miR-520c in U87 cells ( $\mathrm{P}<0.05 ;$ Fig. 3A). Subsequently, miR-520c silencing significantly facilitated the migration and invasion of U87 cells $(\mathrm{P}<0.05$, respectively; Fig. 3B and C). Thus, miR-520c reversed the migratory and invasive abilities of the glioma cells.

TGFBRII is a downstream target of miR-520c. To disclose the underlying molecular mechanisms of the biological function of miR-520c in glioma cells, TargetScanHuman 7.1 (http://www.targetscan.org) was used to search for the downstream target of miR-520c. TGFBRII, a critical regulator of the TGF- $\beta$ pathway in glioma (17), was recognized as a potential downstream target of miR-520c. U251 cells that were transduced with corresponding vectors were subjected to immunoblotting for TGFBRII and CD44, a known downstream target of miR-520c (14). Notably, miR-520c overexpression decreased the levels of TGFBRII and CD44 protein, while miR-520c silencing increased the expression of these proteins (Fig. 4A). Furthermore, qRT-PCR also confirmed that miR-520c overexpression downregulated the mRNA levels of CD44 and TGFBRII ( $\mathrm{P}<0.05$; Fig. 4B). However, miR-520c knockdown showed no significant effect on mRNA expression (Fig. 4B). As shown in Fig. 4C, the 3'-UTR of TGFBRII contained two putative binding sites for miR-520c. Subsequently, we performed luciferase assay to investigate whether miR-520c could bind to the putative binding sites in the 3'-UTR of TGFBRII. Alteration of miR-520c inversely regulated the luciferase activity of TGFBRII $3^{\prime}-\mathrm{UTR}(\mathrm{P}<0.05$; Fig. 4D), while mutant miR-520c did not exhibit any influence on the luciferase activity of TGFBRII 3'-UTR (Fig. 4D). Therefore, these data indicate that TGFBRII is a direct downstream target of miR-520c in glioma.

An inverse correlation between TGFBRII and miR-520c is observed in glioma specimens. Glioma and non-tumor tissues were subjected to qRT-PCR for TGFBRII mRNA. Our data indicated that the expression levels of TGFBRII mRNA in glioma tissues were significantly higher than those in adjacent non-cancerous tissues $(\mathrm{P}<0.05$; Fig. 5A). Spearman's correlation analysis indicated that miR-520c was strongly correlated with TGFBRII mRNA expression in the glioma specimens $(r=-0.910, \mathrm{P}<0.001$; Fig. 5B). Moreover, 
A
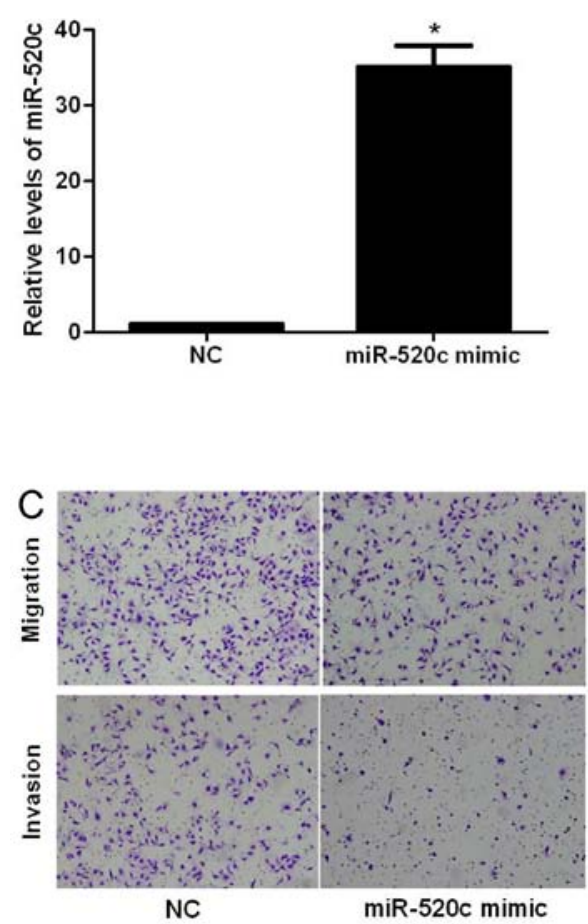

B
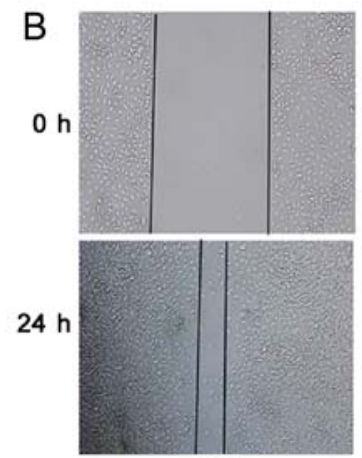

NC
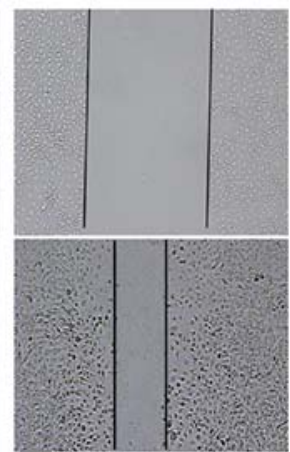

miR-520c mimic

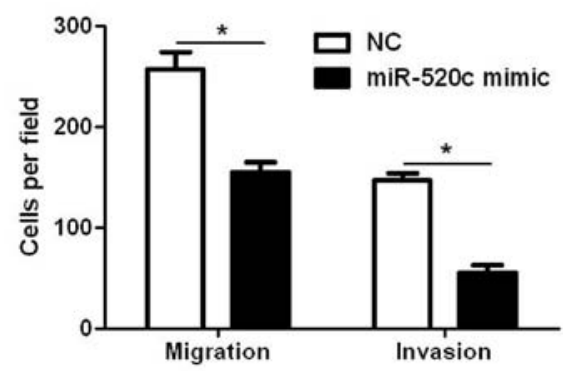

Figure 2. miR-520c mimic inhibits the migration and invasion of U251 cells. (A) miR-520c mimic significantly increased the level of miR-520c expression in U251 cells; "P $<0.05$. (B) miR-520c overexpression reversed the migration of U251 cells. (C) miR-520c overexpression inhibited the migration and invasion of $\mathrm{U} 251$ cells; ${ }^{*} \mathrm{P}<0.05$.

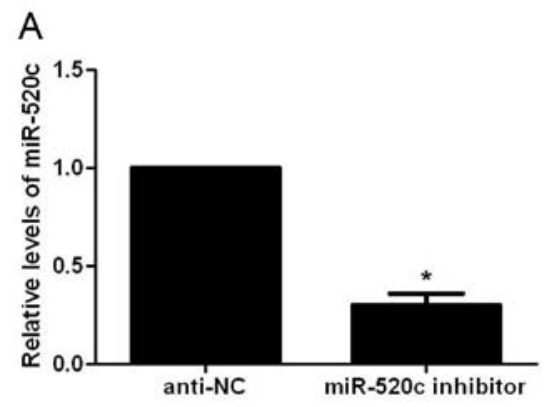

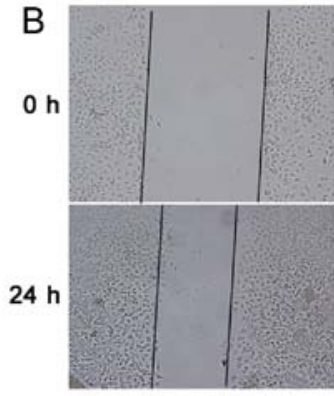

anti-NC

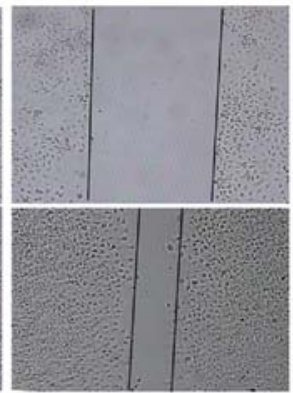

miR-520c inhibitor
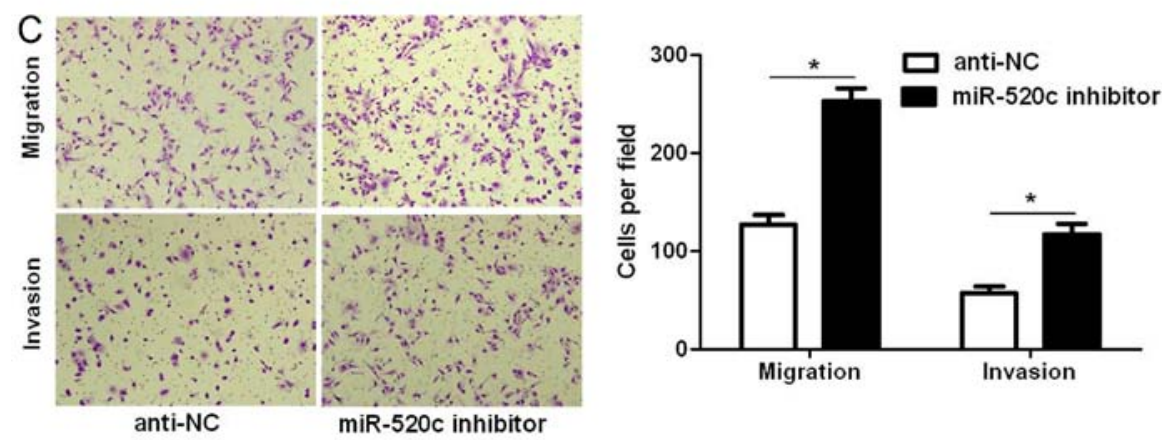

Figure 3. miR-520c inhibitor facilitates the migration and invasion of U87 cells. (A) miR-520c inhibitor significantly decreased the expression of miR-520c in U87 cells; ${ }^{2} \mathrm{P}<0.05$. (B) miR-520c knockdown significantly increased the migration of U87 cells. (C) miR-520c silencing significantly promoted the migration and invasion of $\mathrm{U} 87$ cells; ${ }^{*} \mathrm{P}<0.05$.

immunohistochemical staining indicated that miR-520c highexpressing tumors showed weak staining of TGFBRII, while miR-520c low-expressing tumors showed strong staining of TGFBRII ( $\mathrm{P}<0.05$; Fig. 5C and D).
TGFBRII mediates the functions of miR-520c in glioma cells. Next, U251 cells with miR-520c overexpression were infected with empty vector (EV) or TGFBRII retroviruses. Restoration of TGFBRII expression was confirmed by western 


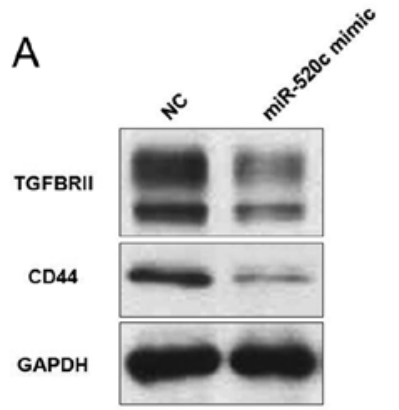

C

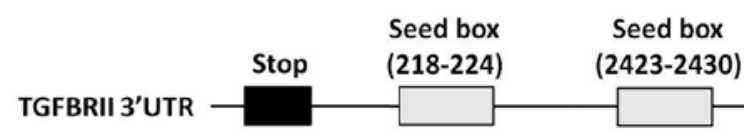

Mature miR-520c-3p

Mutant miR-520c-3p
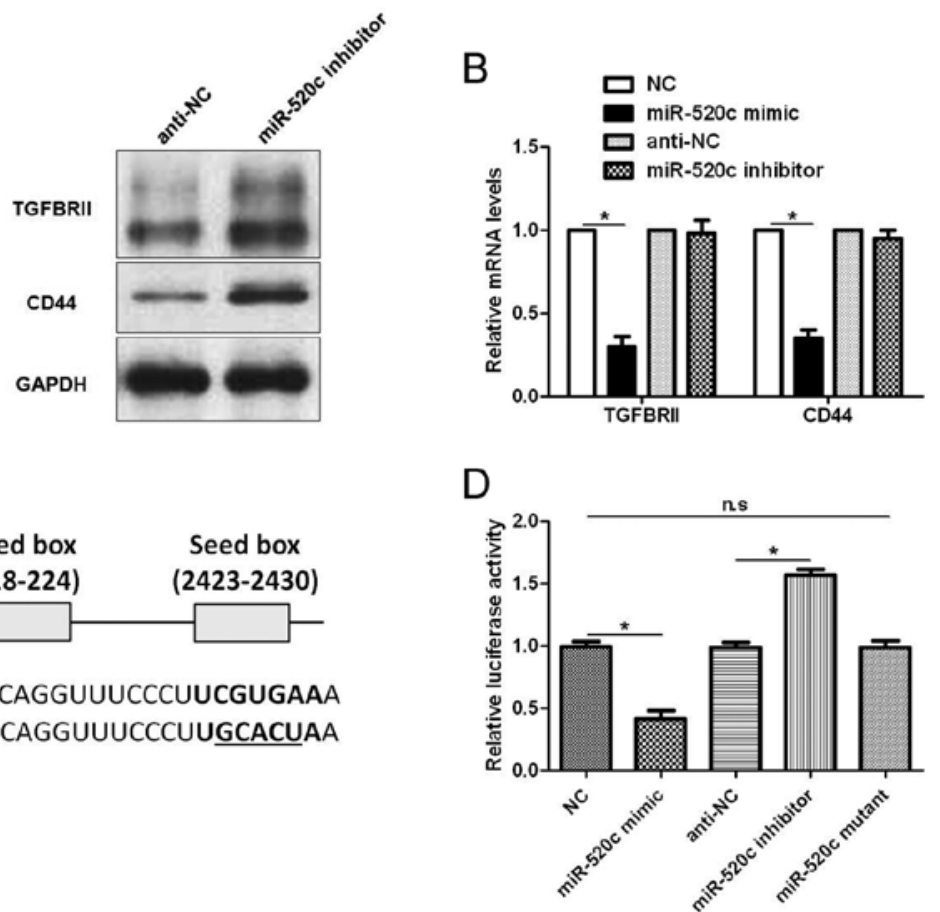

Figure 4. miR-520c binds to the complementary sequence in the 3'-UTR of TGFBRII. (A) miR-520c overexpression reduced while miR-520c silencing increased the level of TGFBRII and CD44 protein in U251 and U87 cells, respectively. (B) miR-520c overexpression decreased the levels of TGFBRII and CD44 mRNA while miR-520c knockdown exhibited no effect on mRNA levels; ${ }^{*} \mathrm{P}<0.05$. (C) The binding sites for miR-520c in 3'-UTR of TGFBRII. (D) Overexpression of miR-520c decreased and miR-520c silencing increased the luciferase activity of the 3'-UTR of TGFBRII, while mutant miR-520c had no effect on the luciferase activity of 3'-UTR of TGFBRII in $293 \mathrm{~T}$ cells; 'P<0.05. n.s., not significantly different.
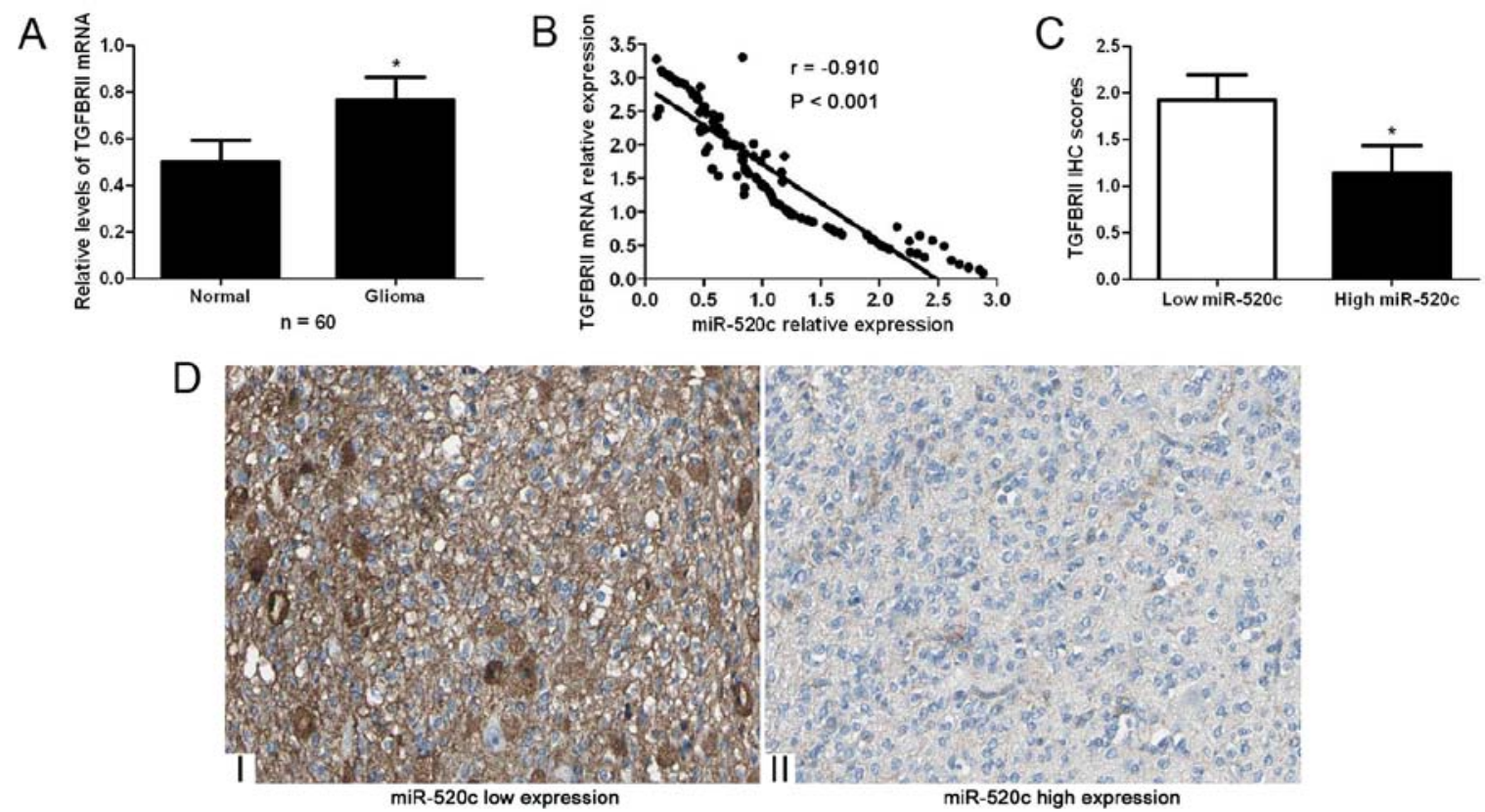

Figure 5. Correlation between TGFBRII and miR-520c in glioma tissues. (A) The expression of TGFBRII mRNA was upregulated in glioma compared to non-tumor tissues; ${ }^{*} \mathrm{P}<0.05$. (B) An inverse correlation between TGFBRII and miR-520c expression was observed in glioma tissues. (C) Quantitative data arising from IHC scores indicated that the expression of TGFBRII in miR-520c low-expressing tumors was evidently higher than that in miR-520c highexpressing cases; ${ }^{*} \mathrm{P}<0.05$. (D) Representative immunohistochemical staining of TGFBRII in glioma tissues. miR-520c high-expressing tumors showed weak staining of TGFBRII (II), while miR-520c low-expressing tumors showed strong staining of TGFBRII (I).

blotting $(\mathrm{P}<0.05$; Fig. 6A). Wound healing and Transwell assays indicated that TGFBRII restoration abrogated the effects of miR-520 coverexpressionin U251 cells withincreased migratory and invasive abilities of glioma cells $(\mathrm{P}<0.05$, respectively;
Fig. 6B and C). Since TGFBRII is a target of miR-520c, and TGFBRII is an important component in TGF- $\beta 1$-induced migration and invasion, miR-520c-overexpressing U251 cells were treated with TGF- $\beta 1(2 \mu \mathrm{g} / \mathrm{ml})$. As expected, the induction 
A
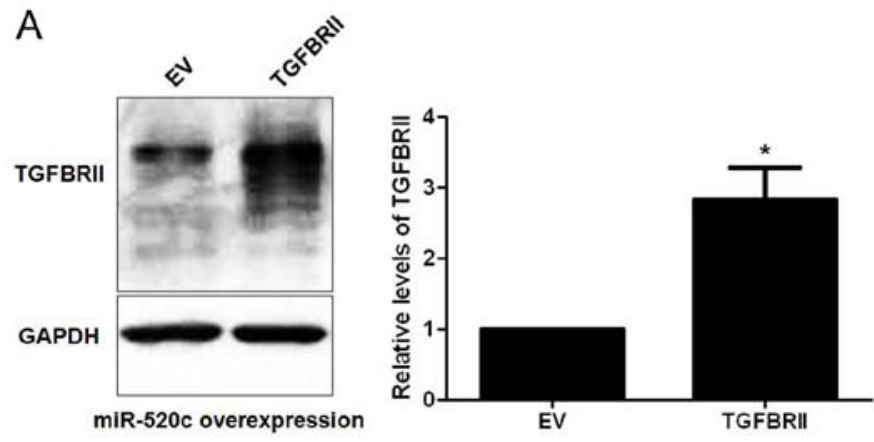

C

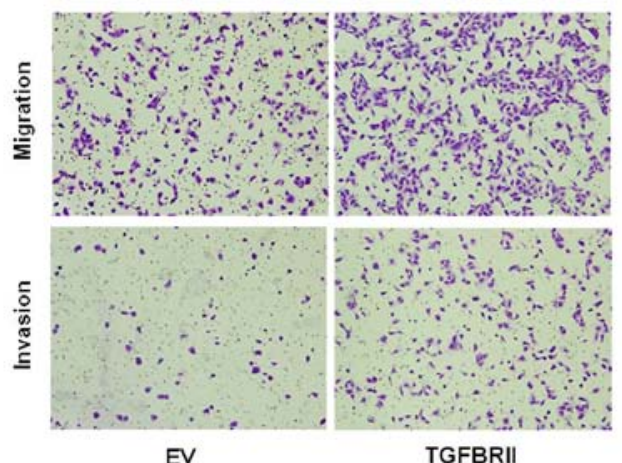

$\mathrm{B}$

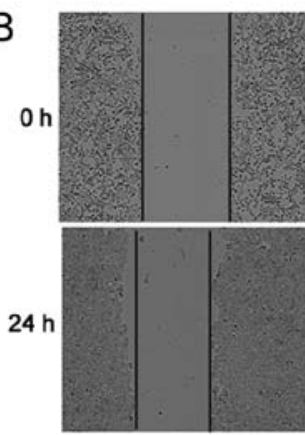

EV
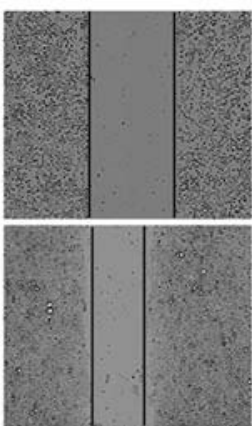

TGFBRII

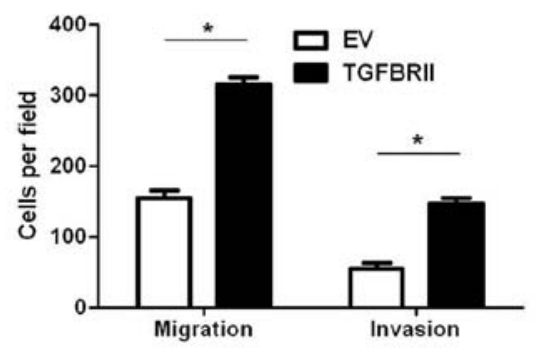

Figure 6. TGFBRII restoration reverses the effects of miR-520c overexpression in U251 cells. (A) miR-520c-overexpressing U251 cells that were transfected with empty vector (EV) or TGFBRII retroviruses were subjected to immunoblotting for TGFBRII; "P<0.05. (B) TGFBRII restoration facilitated the migration of miR-520c-overexpressing U251 cells. (C) TGFBRII restoration promoted the migration and invasion of miR-520c-overexpressing U251 cells; "P<0.05.
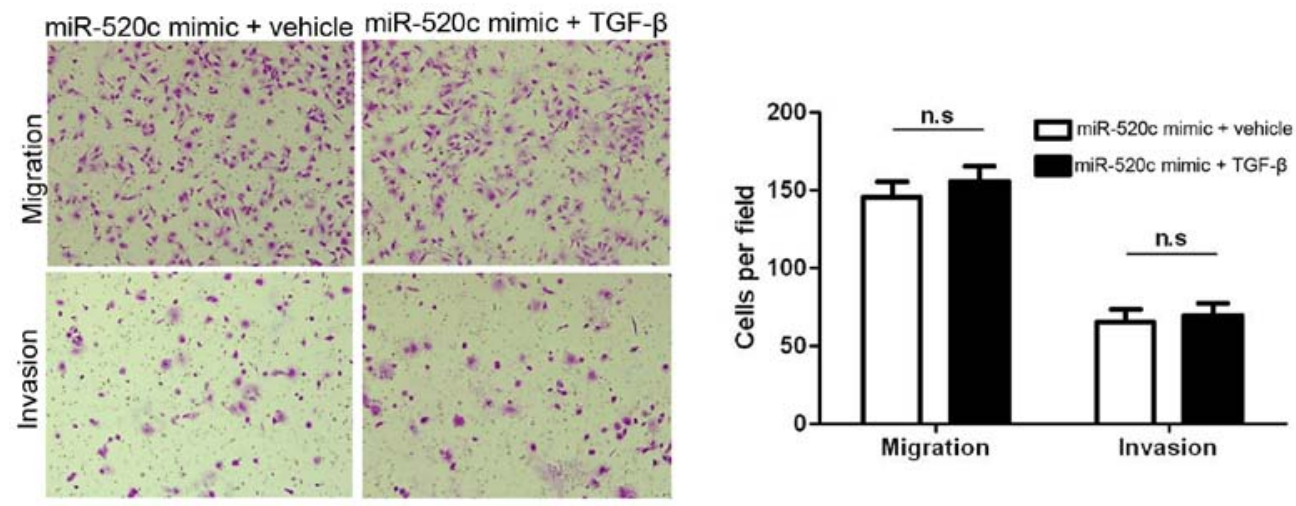

Figure 7. miR-520c overexpression blocks the pro-metastatic effects of TGF- $\beta 1$ on U251 cells. U251 cells that were transfected with miR-520c mimics were treated with vehicle and TGF- $\beta 1(2.5 \mathrm{ng} / \mathrm{ml})$. Transwell assays indicated that miR-520c overexpression blocked TGF- $\beta 1$-induced migration and invasion in U251 cells. n.s., not significantly different.

of TGF- $\beta 1$ showed no effect on miR-520c-overexpressing U251 cells (Fig. 7). Collectively, TGBRII is not only a target but also a functional mediator of miR-520c.

\section{Discussion}

Emerging evidence has confirmed that miRNAs are actively involved in the pathogensis of glioma (18). In addition, miRNAs have been found to be critical regulators of the metastasis and epithelial-mesenchymal transition of glioma cells (19). Due to the important roles of miRNAs in glioma, miRNAs have been proposed as promising biomarkers and therapeutic targets of glioma (20). In the present study, miR-520c was found to be significantly downregulated in glioma tissues. In addition, the low expression of miR-520c in glioma tissues conferred advanced WHO stage. More importantly, low expression of miR-520c was correlated with decreased overall survival of glioma patients. Therefore, miR-520c plays a tumor-suppressive role in glioma and potentially serves as a promising biomarker for the prognosis of glioma patients.

Systemic metastasis is the cause for the unsatisfactory prognosis of glioma patients (21). Increased migratory and invasive abilities of glioma cells underlie the systemic metastasis of glioma (22). Therefore, it is of great importance to elucidate the molecular mechanisms involved in the metastasis of glioma cells. In the present study, we found that 
miR-520c inhibited the migration and invasion of glioma cells in vitro, suggesting that miR-520c exerted an antimetastatic role in glioma. The TGF- $\beta$ signaling pathway has been reported to be implicated in tumor metastasis in various human malignancies (23). The fundamental role of the TGF- $\beta$ pathway in promoting cancer progression in multiple stages of the metastatic process, including epithelial-to-mesenchymal transition (EMT), is also becoming increasingly clear (23). Notably, the TGF- $\beta$-SMAD pathway confers poor prognosis to glioma patients and promotes cell proliferation (24). TGFBRII is a critical component of the TGF- $\beta$ signaling pathway and TGFBRII silencing blocked the effects of TGF- $\beta 1$ in glioma cells (25). In the present study, we found that miR-520c negatively regulated the abundance of TGFBRII in glioma cells. In addition, the expression of TGFBRII in glioma tissues was inversely correlated with miR-520c expression. Moreover, we found that miR-520c directly interacted with the 3'-UTR of TGFBRII using a luciferase reporter assay. These data indicate that TGFBRII is a direct downstream target of miR-520c in glioma. Importantly, restoration of TGFBRII abolished the effects of miR-520c overexpression in glioma cells and miR-520c overexpression blocked TGF- $\beta 1$-induced migration and invasion of glioma cells. Thus, TGFBRII is a functional mediator of miR-520c in glioma.

In conclusion, the present study demonstrated that miR-520c expression was significantly decreased in glioma tissues. Decreased expression of miR-520c was found to be correlated with adverse clinical features and poor prognosis of glioma patients. In addition, miR-520c inhibited migration and invasion of glioma cells. Furthermore, TGFBRII is a direct functional target of miR-520c in glioma. These data may result in the discovery of therapeutic candidates of glioma.

\section{References}

1. Bartel DP: MicroRNAs: Target recognition and regulatory functions. Cell 136: 215-233, 2009.

2. Ambros V: The functions of animal microRNAs. Nature 431: $350-355,2004$

3. Kloosterman WP and Plasterk RH: The diverse functions of microRNAs in animal development and disease. Dev Cell 11: 441-450, 2006

4. Zhang W, Dahlberg JE and Tam W: MicroRNAs in tumorigenesis: A primer. Am J Pathol 171: 728-738, 2007.

5. Esquela-Kerscher A and Slack FJ: Oncomirs - microRNAs with a role in cancer. Nat Rev Cancer 6: 259-269, 2006.

6. Zhang B, Pan X, Cobb GP and Anderson TA: microRNAs as oncogenes and tumor suppressors. Dev Biol 302: 1-12, 2007.

7. Wang $\mathrm{BC}$ and $\mathrm{Ma} \mathrm{J}$ : Role of microRNAs in malignant glioma. Chin Med J 128: 1238-1244, 2015.

8. Wang H, Xu T, Jiang Y, Yan Y, Qin R and Chen J: MicroRNAs in human glioblastoma: From bench to beside. Front Biosci 20 : $105-118,2015$

9. Brower JV, Clark PA, Lyon W and Kuo JS: MicroRNAs in cancer: Glioblastoma and glioblastoma cancer stem cells. Neurochem Int 77: 68-77, 2014.

10. Mazan-Mamczarz K, Zhao XF, Dai B, Steinhardt JJ, Peroutka RJ, Berk KL, Landon AL, Sadowska M, Zhang Y, Lehrmann E, et al: Down-regulation of eIF4GII by miR-520c-3p represses diffuse large B cell lymphoma development. PLoS Genet 10: e1004105, 2014.
11. Keklikoglou I, Koerner C, Schmidt C, Zhang JD, Heckmann D, Shavinskaya A, Allgayer H, Gückel B, Fehm T, Schneeweiss A, et al: MicroRNA-520/373 family functions as a tumor suppressor in estrogen receptor negative breast cancer by targeting NF- $\kappa \mathrm{B}$ and TGF- $\beta$ signaling pathways. Oncogene 31: 4150-4163, 2012.

12. Liu $\mathrm{P}$ and Wilson MJ: $\mathrm{miR}-520 \mathrm{c}$ and $\mathrm{miR}-373$ upregulate MMP9 expression by targeting mTOR and SIRT1, and activate the Ras/Raf/MEK/Erk signaling pathway and NF- $\mathrm{KB}$ factor in human fibrosarcoma cells. J Cell Physiol 227: 867-876, 2012.

13. Toffanin S, Hoshida Y, Lachenmayer A, Villanueva A Cabellos L, Minguez B, Savic R, Ward SC, Thung S, Chiang DY, et al: MicroRNA-based classification of hepatocellular carcinoma and oncogenic role of miR-517a. Gastroenterology 140: 1618-1628.e16, 2011

14. Huang Q, Gumireddy K, Schrier M, le Sage C, Nagel R, Nair S, Egan DA, Li A, Huang G, Klein-Szanto AJ, et al: The microRNAs miR-373 and miR-520c promote tumour invasion and metastasis. Nat Cell Biol 10: 202-210, 2008.

15. Lu S, Zhu Q, Zhang Y, Song W, Wilson MJ and Liu P: Dual-functions of miR-373 and miR-520c by differently regulating the activities of MMP2 and MMP9. J Cell Physiol 230: 1862-1870, 2015.

16. Tu K, Li J, Verma VK, Liu C, Billadeau DD, Lamprecht G, Xiang X, Guo L, Dhanasekaran R, Roberts LR, et al: Vasodilator-stimulated phosphoprotein promotes activation of hepatic stellate cells by regulating Rab11-dependent plasma membrane targeting of transforming growth factor $\beta$ receptors. Hepatology 61: 361-374, 2015.

17. Ye XZ, Xu SL, Xin YH, Yu SC, Ping YF, Chen L, XiaoHL, Wang B, Yi L, Wang QL, et al: Tumor-associated microglia/macrophages enhance the invasion of glioma stem-like cells via TGF- $\beta 1$ signaling pathway. J Immunol 189: 444-453, 2012.

18. Xue H, Gao X, Xu S, Zhang J, Guo X, Yan S, Li T, Guo X, Liu Q and Li G: MicroRNA-Let-7f reduces the vasculogenic mimicry of human glioma cells by regulating periostin-dependent migration. Oncol Rep 35: 1771-1777, 2016.

19. Li J, Yuan J, Yuan X, Zhao J, Zhang Z, Weng L and Liu J: MicroRNA-200b inhibits the growth and metastasis of glioma cells via targeting ZEB2. Int J Oncol 48: 541-550, 2016.

20. Fan B, Jiao BH, Fan FS, Lu SK, Song J, Guo CY, Yang JK and Yang L: Downregulation of miR-95-3p inhibits proliferation, and invasion promoting apoptosis of glioma cells by targeting CELF2. Int J Oncol 47: 1025-1033, 2015.

21. Usinskiene J, Ulyte A, Bjørnerud A, Venius J, Katsaros VK, Rynkeviciene R, Letautiene S, Norkus D, Suziedelis K, Rocka S, et al: Optimal differentiation of high- and low-grade glioma and metastasis: A meta-analysis of perfusion, diffusion, and spectroscopy metrics. Neuroradiology 58: 339-350, 2016

22. Liu J, Zhang J, Huang L, Zhu X, Chen W and Hu P: XuefuZhuyu Tang exerts antitumor effects by inhibiting glioma cell metastasis and invasion via regulating tumor microenvironment. Onco Targets Ther 9: 3603-3612, 2016.

23. Papageorgis P: TGF $\beta$ Signaling in tumor initiation, epithelial-tomesenchymal transition, and metastasis. J Oncol 2015: 587193, 2015.

24. Bruna A, Darken RS, Rojo F, Ocaña A, Peñuelas S, Arias A, Paris R, Tortosa A, Mora J, Baselga J, et al: High TGF $\beta$-Smad activity confers poor prognosis in glioma patients and promotes cell proliferation depending on the methylation of the PDGF-B gene. Cancer Cell 11: 147-160, 2007.

25. Wei F, Wang Q, Su Q, Huang H, Luan J, Xu X and Wang J: miR-373 inhibits glioma cell U251 migration and invasion by down-regulating CD44 and TGFBR2. Cell Mol Neurobiol 36: 1389-1397, 2016. 